\title{
THERMAL BEHAVIOUR OF ESR SIGNALS OBSERVED IN VARIOUS NATURAL CARBONATES
}

\author{
Jean-Jacques Bahain, Yuji Yokoyama, Hassan Masaeudi, Christophe Falguères and Michel Laurent \\ Institut de Paléontologie Humaine, 1, rue René Panhard, 75013 Paris, France
}

\begin{abstract}
The thermal behaviour of the five main ESR lines observed in Quaternary carbonates $(g=2.0057, g=2.0031$, $g=2.0018, g=2.0006$ and $g=1.9972$ ) was studied through isochron annealing experiments. As the general rule, ESR lines are more stable in calcitic samples than in aragonitic ones and in speleothems than in marine carbonates. In all the studied carbonates, the most stable signals are $g=2.0057$ and $g=2.0031$, then $g=2.0006$.
\end{abstract}

\section{INTRODUCTION}

Since the first attempt to date a stalactite by ESR (Ikeya, 1975), natural carbonates have been the object of many studies. Indeed these minerals are very common in Quaternary deposits in various forms (speleothems, travertines, corals, foraminifera, mollusc shells, etc.) and may contain important information about palaeoclimatology, palaeogeography or archaeological chronology. Today, the nature of the most paramagnetic species observed in Quaternary carbonates is known with respect to structure, ESR features, dose-response, thermal behaviour, stability etc. (see for example Grün, 1989; Barabas et al., 1992a, b; Bahain, 1993), but it is rather difficult to compare results obtained in different works, because, on the one hand, they have been generally focused on a specific kind of carbonates (often calcitic speleothems or aragonitic corals), and, on the other hand, experimental procedures may vary greatly from one study to another, especially concerning annealing experiments.

The main purpose of the present work is to observe and compare the evolution of ESR spectra for different carbonates during cumulated annealing experiments, in order to measure the effect of the nature, the mineralogy, the origin or the geological history of the samples on the thermal behaviour of the main ESR lines.

\section{ESR SPECTRA OF CARBONATES}

Six types of fossil carbonates have been studied: calcitic and aragonitic speleothems, aragonitic and calcitic (recrystallised) corals and aragonitic and calcitic (non-recrystallised) mollusc shells. The natural ESR spectra of these different carbonates are shown in Fig. 1.

ESR spectra of calcitic speleothems can change considerably from one sample to another (see for example Grün, 1989), but they show generally several isotropic lines at $g=2.0057, g=2.0040, g=2.0031$ and $g=2.0006$, and a big rhombic signal at $g_{x x}=2.0030$, $g_{z z}=2.0015, g_{y y}=1.9972$. Spectra can be complicated by peaks due to paramagnetic impurities $\left(\mathrm{Mn}^{2+}\right.$ and/or $\mathrm{Fe}^{3+}$ ), irradiation (short-living signal, $g=2.0023$ ) or grinding (crushing signal, $g=2.0001$ ). Several lines have been used for dating ( $g=2.0057, g=2.0040$ and $g=2.0006$ ) but even today the choice of the dating signal is the subject of controversy. On the other hand, relatively little is known about aragonitic speleothems. The ESR spectrum of the sample used in this study is nearly similar to the calcitic one, except for a very poor peak resolution. Lines at $g=2.0057$ and 2.0006 are present as well as the rhombic signal. This latter one has been used for dating (see Grün, 1989).

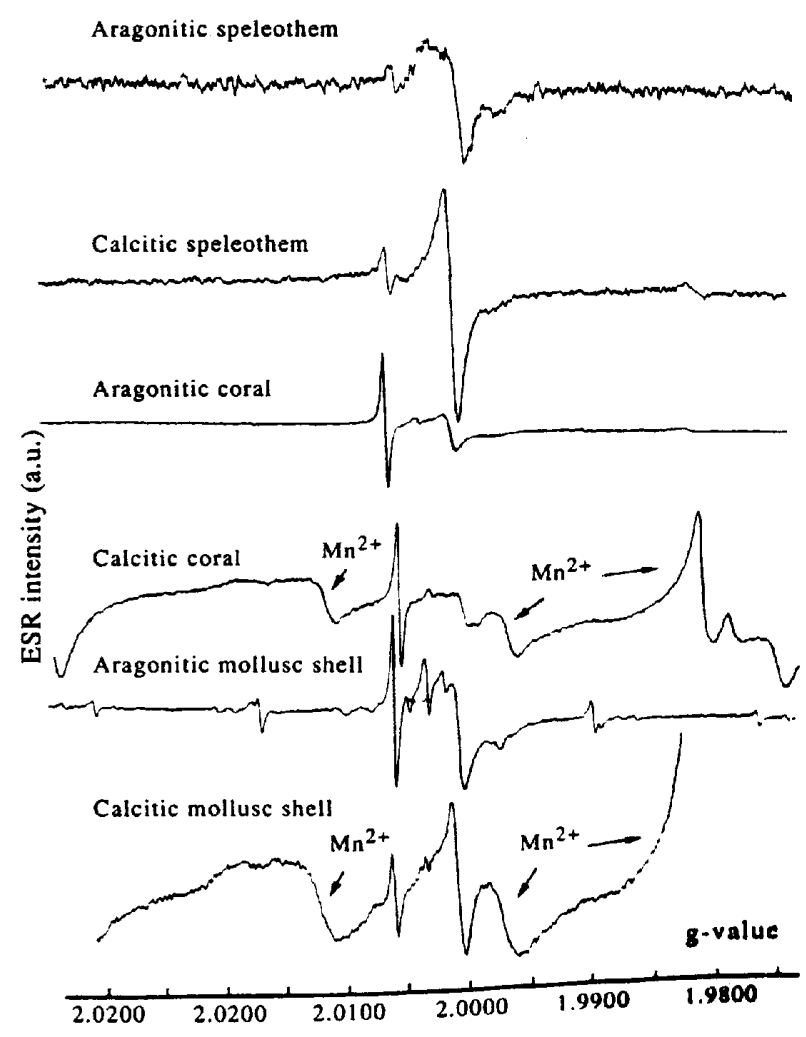

FIG. 1. Natural ESR spectra of several Quaternary carbonatcs. 
ESR spectra of aragonitic corals display three peaks at $g=2.0057, g=2.0031$ and $g=2.0006$ (the latter is the dating signal). In the sample used in this study, the rhombic signal with a gap at $g=1.9972$ and an isotropic line at $g=2.0018$ appear with irradiation. These signals are also present in recrystallised corals as well as parasitic peaks (crushing signal and/or $\mathrm{Mn}^{2+}$ lines).

Mollusc shells show complex ESR spectra and five main lines $(g=2.0057, g=2.0018, g=2.0006$, and the rhombic one) and additionally paramagnetic impurities signals. At present, there is no concensus about the choice of a useful dating signal.

The main ESR features of signals observed in Quaternary carbonates are summarized in Barabas (1992).

Five signals are often present in natural carbonates: $g=2.0057$ (called $h-1$ by Yokoyama et al., 1981); $g=2.0031$ (or $g_{\perp}=2.0036$ and $\left.g \|=2.0021\right)(b$, Ikeya and Ohmura, 1981); $g=2.0018$ ( $g$-3, Molodkov, 1988); $g=2.0006(h-3$, Yokoyama et al., 1981); $g=1.9972$ $\left(g_{x x}=2.0030, g_{z z}=2.0015, g_{y y}=1.9972\right)$ (A-complex, Barabas et al., 1992a). The thermal behaviour of these five lines are studied in this work.

\section{EXPERIMENTAL}

The resolution of the ESR spectrum of the aragonitic speleothem sample is not very good because of its young age. On the other hand, some ESR signals appear only after irradiation. So, for each sample, five aliquots were irradiated with a ${ }^{60} \mathrm{Co}$-source (at 42.5 , $97.5,172.5,380$ and $570 \mathrm{~Gy}$ ). The thermal evolution was studied from a mean value of the ESR intensities, which corresponds to the non-irradiated and the five irradiated aliquots' intensities.

Powders (100-200 $\mu \mathrm{m}$ size) were filled in Suprasil tubes ( $3 \mathrm{~mm}$ inner diameter) and ESR measurements were carried out at room temperature using a Varian E-109 X-band spectrometer. The experimental parameters were: microwave power $-5 \mathrm{~mW}$, modulation amplitude $-0.05 \mathrm{mT}$, scan range $-331 \pm 5 \mathrm{mT}$, time constant $-0.128 \mathrm{sec}$, modulation frequency $-100 \mathrm{kHz}$. The peak-to-peak amplitudes of each signal were measured for each step of temperature, except for the line at $g=1.9972$ for which the distance from the peak to the baseline was used, then the intensities were normalized for weight and height of the powder inside the tube.

The thermal experiments consist of isochron annealings of $16 \mathrm{hr}$ from $80^{\circ} \mathrm{C}$ to $220^{\circ} \mathrm{C}$ with steps of $20^{\circ} \mathrm{C}$, performed with an Adamel T-2 electrical oven. A long annealing time was preferred because the equivalent short annealing at a higher temperature could cause an effect due to the relative thermal unstability of the samples. The effective temperature during annealing was measured continuously and, except for fluctuations at the beginning of the experiments, temperature
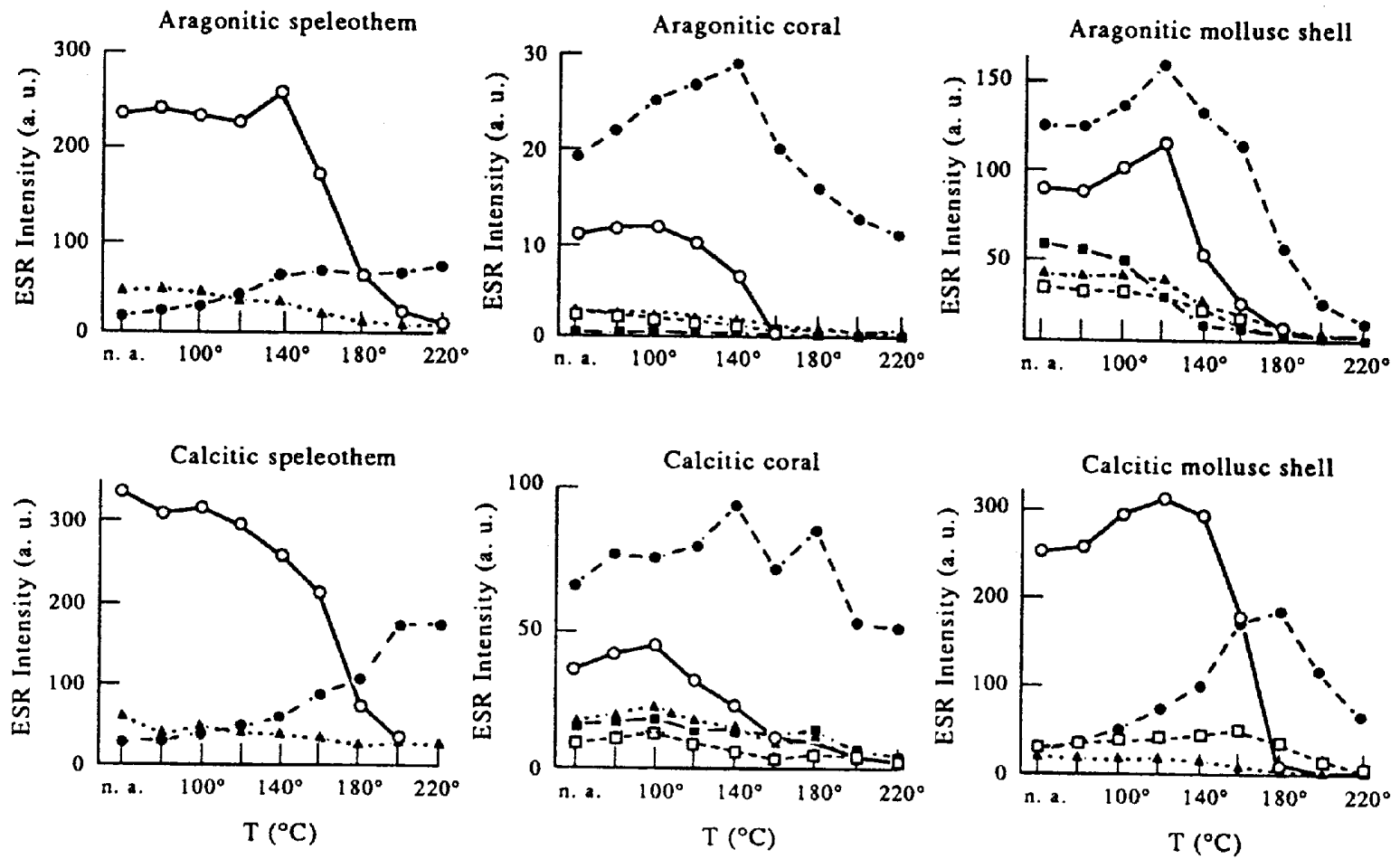

FIG. 2. Effect of cumulated isochron annealing experiments of $16 \mathrm{hr}$ on ESR signals for different carbonates. 
variations did not exceed $\pm 1^{\circ} \mathrm{C}$. As samples were annealed directly inside ESR tubes, in order to reduce differences between two steps of thermal treatment, annealing effects are cumulated.

\section{RESULTS}

Isochron curves obtained for the various carbonates are shown in Fig. 2. In each diagram of this figure, the ratio of the intensities between the different ESR lines observed have been represented. Some interesting observations can be made about these curves.

(1) As a general rule, ESR signals are more stable in calcitic samples than in aragonitic ones.

(2) The general thermal behaviour of the main ESR signals does not change significantly with the mineralogy for speleothems and corals, when curves obtained for mollusc shells are very different according to whether the shell is calcite or aragonite.

(3) Aragonitic corals and aragonitic shells show approximately the same thermal behaviour: a small growth of intensities of the lines at $g=2.0057$ and $g=2.0006$ in the first steps of the thermal treatment, then a quick decrease. These two signals are more stable in aragonitic speleothem.

(4) The diagram obtained for calcitic speleothem is similar to that reported by Hennig and Grün (1983) or Smith et al. (1985). After a relative stability up to $120^{\circ} \mathrm{C}$, the intensity of the line at $g=2.0006$ decreases rapidly, when, in the same time, the signal at $g=2.0057$ is increasing. These features were also observed by Yokoyama et al. (1983), who explained these phenomena by a transfer of electrons from $g=2.0006$ 'to $g=2.0057$. This theory was, however, much criticized in the past (see for example Hennig and Grün, 1983 or Grün, 1989). In the present work, a similar thermal behaviour is also observed for the calcitic mollusc shell while the secondary calcite of recrystallised corals shows thermal features which are nearest to those observed in the aragonitic samples than in the other calcitic carbonates. In this latter case, the nature of the carbonate seems to play a more prominent part on the thermal behaviour than the mineralogy.

(5) ESR signals can be classified in terms of thermal stability:

$$
\begin{aligned}
& g=2.0057>g=2.0031 \geqslant g=2.0006>g=2.0018 \approx \\
& g=1.9972
\end{aligned}
$$

This classification is observed in all the studied samples, but the stability of the ESR centres may change with the nature and the mineralogy of the carbonates.

Figure 3 shows the thermal behaviour of a given ESR signal in the different carbonates. Here, the real sizes of the signals are not respected and only the shape of the isochron curves should be taken into consideration. The following observations can be noted.

(1) The isochron curves of $g=2.0018, g=2.0006$ and $g=1.9972$ are approximately the same for all samples. The intensities of these signals decrease quickly from the very first steps of annealing. The line at $g=2.0006$
2,0057

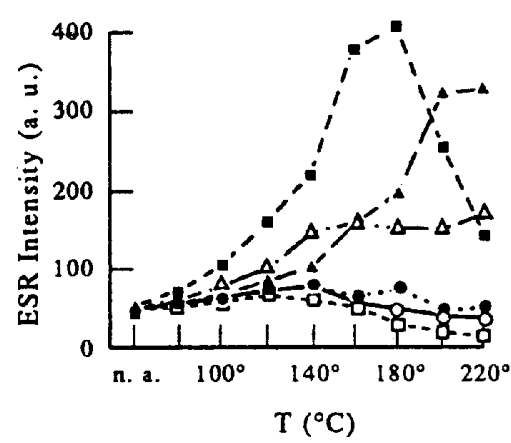

2,0031

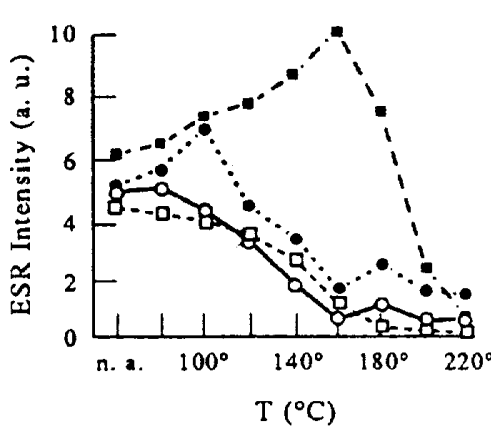

2,0018

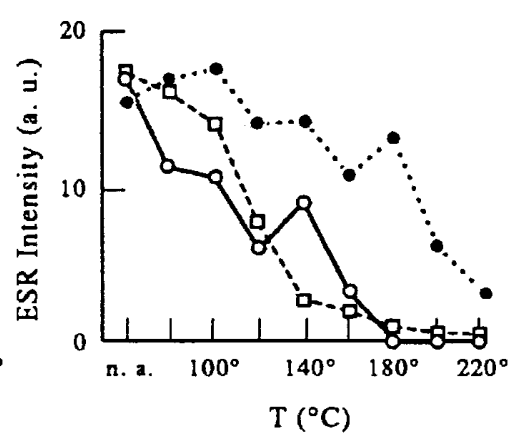

2,0006

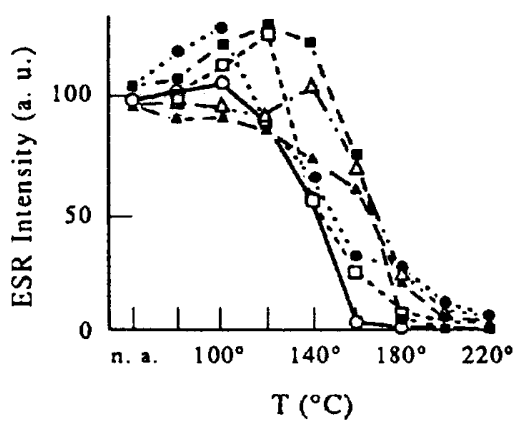

1,9972
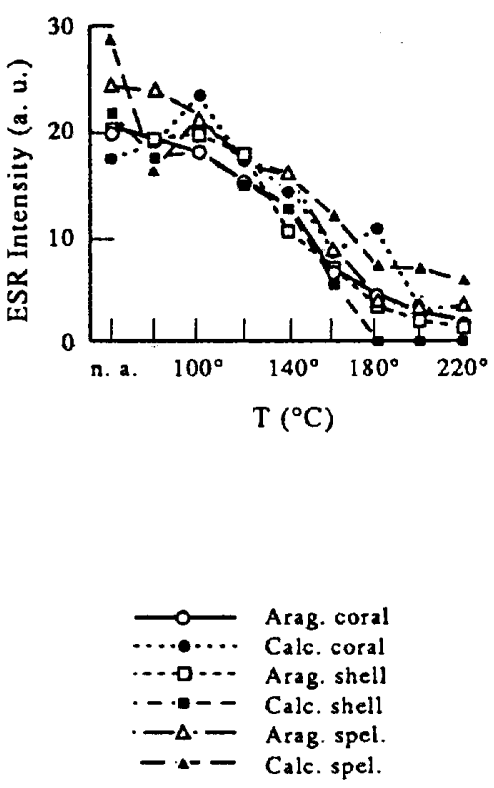

-....... Aarg. coral

...... Arag. shell

- $\Delta \cdot$ - Arag. spel.

- - Calc. spel.

FIG. 3. Effect of cumulated isochron annealing experiments of $16 \mathrm{hr}$ on main ESR signals according to the nature and the mineralogy of the studied carbonates. 
is more stable up to $120-140^{\circ} \mathrm{C}$, then it decreases rapidly and disappears about $180-200^{\circ} \mathrm{C}$.

(2) The thermal behaviour of the signal at $g=2.0031$ seems to be very dependent on the nature and the mineralogy of the studied carbonate. In calcitic marine carbonates, this line shows a low thermal stability, when in calcitic ones, its intensity increases in the first steps of annealing. In calcitic mollusc shell, this initial growth is particularly important and continues up to $160^{\circ} \mathrm{C}$, then intensity decreases quickly.

(3) Two kinds of isochron curves can be distinguished for the line at $g=2.0057$ according to the nature of the carbonates. In corals and in aragonitic mollusc shell, the signal is stable or grows slightly up to $120-140^{\circ} \mathrm{C}$ then decreases. On the other hand, in speleothems and in calcitic shell, the intensity of $g=2.0057$ strongly increases up to $160-200^{\circ} \mathrm{C}$ up to maximum values, which can be 8-10 times higher than the initial intensity. The mineralogy and the biological or geological nature of the carbonates have therefore a considerable importance on the stability of the paramagnetic centre responsible for this line.

\section{CONCLUSION}

Isochron annealing experiments show that the thermal behaviour of the main ESR signals observed in natural carbonates greatly changes with the nature and the mineralogy of the samples. As a general rule, signals are more stable in calcitic samples than in aragonitic ones and in speleothems than in marine carbonates. It is also possible to classify ESR signals in terms of thermal stability: the lines at $g=2.0057$ and $g=2.0031$ are the most stable, then $g=2.0006$.

The relative instability of the line at $g=2.0006$ is probably related to the fact that the $\mathrm{CO}_{2}-$ radicals responsible for this signal are associated with water molecules (Barabas et al., 1992a; Debuyst et al., 1993). Moreover, intensity measurements of this line are often difficult because of the presence of the very unstable A-complex signal. The low thermal stability of the line at $g=2.0006$ was already discussed (Yokoyama et al., 1983; Barabas et al., 1992b).

The signal at $g=2.0031$ shows a good thermal stability in calcitic mollusc shell. This fact can be put together with the works of Barabas et al. (1988) and Mudelsee et al. (1992), in which this signal is used to date calcitic foraminifera. On the other hand, in aragonitic marine carbonates, this line displays a very poor thermal stability and its use for dating does not seem possible.

The use of the line at $g=2.0057$ to date speleothems is a subject of controversy, on account of the non$\gamma$-sensibility of this signal and to its growth during thermal treatments (see, for example Grün, 1989; Bahain, 1993). An increase of the signal intensity is also observed during the thermal treatment of the calcitic mollusc shell, but not in aragonitic or recrystallized samples. This phenomenon could be related to a structural feature of the calcite rather than to the nature of the paramagnetic centre responsible for the signal. However, the present study confirms that this line is the most stable signal observed in Quaternary natural carbonates.

\section{ACKNOWLEDGEMENTS}

We gratefully acknowledge B. Morin (Laboratoire de Spectrochimie, Université de Paris VI) for his help during ESR measurements. Thanks to B. Keraudren, J.-L. Monnier and $G$. Giacobini for providing the samples and $R$. Grün for correcting the manuscript.

\section{REFERENCES}

Bahain, J.J. (1993). Datation par résonance de spin électronique (ESR) de carbonates et d'émail dentaire quaternaires: potentiel et problèmes. Unpublished Ph.D. Thesis, Paris, 114 pp.

Barabas, M. (1992). The nature of the paramagnetic centres at 2.0057 and 2.0031 in marine carbonates. Nuclear Tracks and Radiation Measurements, 20, 453-464.

Barabas, M., Bach, A. and Mangini, A. (1988). An analytical model for ESR-signals in calcite. Nuclear Tracks and Radiation Measurements, 14, 231-235.

Barabas, M., Bach, A., Mudelsee, M. and Mangini, A. (1992a). General properties of the paramagnetic centre at $g=2.0006$ in carbonates. Quaternary Science Reviews, 11, $165-171$.

Barabas, M., Mudelsee, M., Walther, R. and Mangini, A. (1992b). Dose-response and thermal behaviour of the ESR signal at $g=2.0006$ in carbonates. Quaternary Science Reviews, 11, 173-179.

Debuyst, R., Dejehet, F. and Idrissi, S. (1993). Paramagnetic centers in $\gamma$-irradiated synthetic monohydrocalcite. Applied Radiation and Isotopes, 44, 293-297.

Grün, R. (1989). Electron spin resonance (ESR) dating. Quaternary International, 1, 65-109.

Hennig, G. and Grün, R. (1983). ESR dating in Quaternary geology. Quaternary Science Reviews, 2, 157-238.

Ikeya, M. (1975). Dating a stalagmite by electron paramagnetic resonance. Nature, 255, 48-50.

Ikeya, M. and Ohmura, K. (1981). Dating of fossil shells with electron spin resonance. Journal of Geology, 89, 247-251.

Molodkov, A. (1988). ESR dating of Quaternary shells: Recent advances. Quaternary Science Reviews, 7, 477-484.

Mudelsee, M., Barabas, M. and Mangini, A. (1992). ESR dating of the Quaternary deep-sea sediment core RC17177. Quaternary Science Reviews, 11, 181-189.

Smith, B.W., Smart, P.L. and Symons, M.C.R. (1985). ESR signals in a variety of speleothem calcites and their suitability for dating. Nuclear Tracks and Radiation Measurements, 10, 837-844.

Yokoyama, Y., Quaegebeur, J.P., Bibron, R., Léger, C., Nguyen, H.V. and Poupeau, G. (1981). Electron spin resonance (ESR) dating of stalagmites of the Caune de l'Arago at Tautavel. In: de Lumley, H. and Labeyrie, J. (eds), Absolute Dating and Isotope Analysis in Prehistory - Methods and Limits, pp. 507-532. Proceedings, Éditions due C.N.R.S., Paris.

Yokoyama, Y., Quaegebeur, J.P., Bibron, R. and Léger, C. (1983). ESR dating of paleolithic calcite: thermal annealing

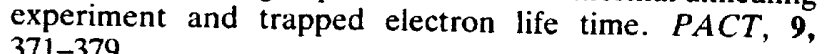

